Check for updates

Cite this: RSC Adv., 2019, 9, 30381

Received 10th July 2019

Accepted 6th September 2019

DOI: $10.1039 / c 9 r a 05255 k$

rsc.li/rsc-advances

\section{Side chain effects on the solid-state emission behaviours and mechano-fluorochromic activities of $10 \mathrm{H}$-phenothiazinylbenzo[d] imidazoles $\dagger$}

\begin{abstract}
Hao Jiang, Xiao-Jing Liu, Ran-Rong Jia, Tian-Hui Xu and Min Xia (D)*
A family of 4-cyanophenyl-substituted $10 \mathrm{H}$-phenothiazinylbenzo[d]imidazoles with different side chains at the 10-position are prepared and their physical properties are studied. The detailed structure-property research demonstrates that the cold crystallization temperature of ground samples and the emission wavelengths of pristine samples are in good accordance with the packing density, conformation distortion and intermolecular interactions, but emission wavelengths of ground samples are slightly chain-dependent. For benzimidazoles with alkyl chains, longer and more branched chains can produce looser packings, which cause pristine samples to display red-shifted emission and reduced MFC activity. For benzimidazoles with a phenyl chain, the emission wavelengths of both the pristine and the ground samples are remarkably red-shifted. Moreover, the degree of conformation distortion is larger, and the cold crystallization temperature is higher. Interestingly, the homologue with the $n$-hexyl chain displays an intense $M L$ effect that is mainly attributed to the heavy discharge quantity on largely enhanced discharge areas under force stimuli due to the great fragility of this crystal.
\end{abstract}

\section{Introduction}

Recently, mechano-fluorochromic (MFC) compounds, ${ }^{1}$ which are smart materials that are capable of making reversible and dynamic responses to external force stimuli by producing varied emission wavelengths or intensities, have increasingly become a hot topic in the field of material chemistry. This is due to their outstanding performances in pressure or chemical sensors, ${ }^{2}$ memory chips, ${ }^{3}$ security inks ${ }^{4}$ or papers, ${ }^{5}$ and optical data storage devices. ${ }^{6}$ Such solid-state photophysical properties are highly sensitive to the conformation or/and packing modes. Since chains can effectively influence intermolecular interactions, packing modes and molecular conformations through the control of the chain length or the degree of branching, sidechain engineering has been a practical and convenient method for successfully tuning the MFC performances of many systems in recent years. ${ }^{7}$ However, the side chain effect is very complicated and emission wavelength shifts in response to force stimuli are uncertain. If side chains can effectively separate molecules from each other to generate a large enough free volume in which molecules adopt more twisted conformations,

Department of Chemistry, Zhejiang Sci-Tech University, Hangzhou 310018, P. R. China.E-mail:xiamin@zstu.edu.cn

$\dagger$ Electronic supplementary information (ESI) available: ${ }^{1} \mathrm{H}$ and ${ }^{13} \mathrm{C}$ NMR spectra, absorption and emission spectra, PXRD patterns, DSC curves, X-ray crystallography and others. CCDC 1919632-1919637, 1919652. For ESI and crystallographic data in CIF or other electronic format see DOI: $10.1039 / \mathrm{c} 9 \mathrm{ra05255k}$ a positive effect will be produced with blue-shifted emission in the pristine state and improved MFC activity. In contrast, more free volume due to side chain extension may cause the conformations to be more planar, and a negative effect will be presented with red-shifted emission in the as-prepared state along with weakened MFC activity. When conformations cause slight changes in side chain separation, the force-induced shifts will be slightly sensitive to different alkyl chain lengths. In literature, the cases with positive ${ }^{8}$ or negative ${ }^{9}$ or small side effects ${ }^{10}$ have been reported. Occasionally, emission shifts before and after force application are firstly increased and then decreased, ${ }^{\mathbf{1 1}}$ or vice versa,${ }^{\mathbf{1 2}}$ as alkyl chains are extended. Hence, for a given fluorophore and its homologues, the side chain effect on their MFC performances is difficult to predict and should be investigated individually. So far, for most reports on the side chain effect, only experimental results have been presented and detailed structure-property studies in view of molecular conformations and intermolecular interactions are seldom carried out. In this work, deep insight into the relationship between side chains and MFC behaviours will be afforded for a family of homologues by means of X-ray crystallography and theoretical calculations.

Recently, substituted imidazoles have been reported to be MFC-active systems. ${ }^{13}$ Most cases are focused on polycyclic fused 1,2-diarylimidazoles, ${ }^{\mathbf{1 3 b}}$ while only one report on 1,2-diarylbenzo[d]imidazoles can be tracked in literature. In order to extend the research in this field, a family of 1,2-diarylbenzo[ $d]$ imidazoles, with 4-cyanophenyl and phenothiazine (PTZ) as the electron-donating and electron-withdrawing group, 
respectively, has been conveniently obtained by the introduction of different side chains at the 10-position on PTZ. PTZ is a unique building block with a high HOMO level and a bowlshaped conformation, which is endowed with the capability of displaying good performance in thermally activated delayed fluorescence, ${ }^{14}$ aggregation-induced emission ${ }^{15}$ and the MFC effect. ${ }^{16}$ The vast majority of reports on the chain effect have only considered straight-chain alkyl groups, and little attention has been paid to branched alkyls, ${ }^{17}$ while aryl chains have never been considered. Herein, we have conducted a systematic study on the MFC behaviours of 1,2-diarylbenzo[d]imidazoles (BIMP) having unbranched and branched alkyl chains along with a phenyl chain on the PTZ ring.

\section{Results and discussion}

\section{Synthesis}

The BIMP members can be readily obtained in good yields via a three-step process (Fig. 1). The aromatic nucleophilic substitution takes place between 2-fluoronitrobenzene and 4-cyanoaniline to produce 4-(2-nitrophenyl amino)benzonitrile 1 in $77 \%$ yield as an orange solid. 4-(2-Aminophenyl amino)benzonitrile 2 was obtained in $88 \%$ yield in the presence of hydrated hydrazine under the catalysis of $\mathrm{Pd} / \mathrm{C}$. Such reduction displays the obvious colour change from orange to greyish white. The subsequent cyclization can be smoothly carried out in $64-87 \%$ yield by the condensation of 2 and $\mathrm{N}$-substituted $10 \mathrm{H}^{-}$ phenothiazine-3-carbaldehyde in DMSO under an air atmosphere. For this cyclization, DMSO acts as the oxidant and it is better to carry out this reaction in an open vessel in order to accelerate the rate. All the products and the intermediates were determined by ${ }^{1} \mathrm{H}$ and ${ }^{13} \mathrm{C}$ NMR spectroscopy to obtain satisfactory data (ESI $\dagger$ ). All the BIMP homologues are solids with intense emission under UV light irradiation and their crystals are easily obtained by recrystallization from $n$-hexane $/ \mathrm{CHCl}_{3}$ mixture.

\section{Photophysical properties in solution}

$\mathbf{B I M P}_{2}$ was selected to investigate its absorption and emission behaviors in solution, and the corresponding spectra are presented in Fig. 2A. The absorption wavelength $\left(\lambda_{\mathrm{ab}}\right)$ maxima are

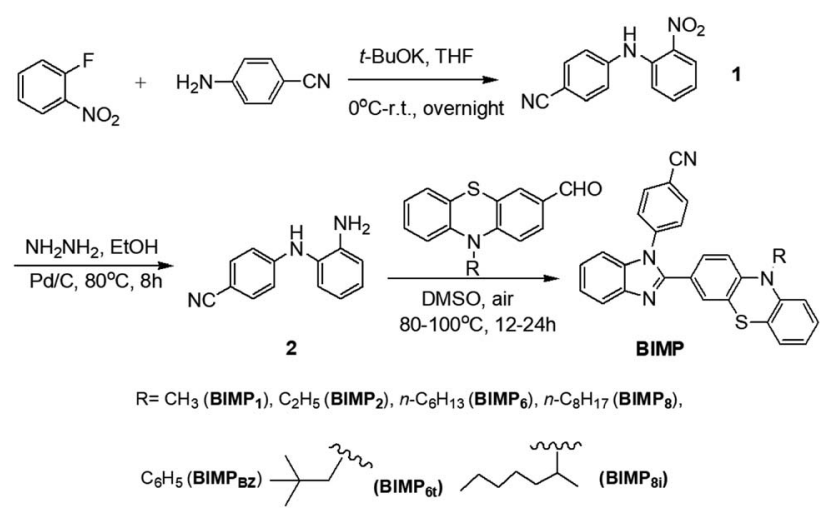

Fig. 1 Synthetic route to the title compounds BIMP.
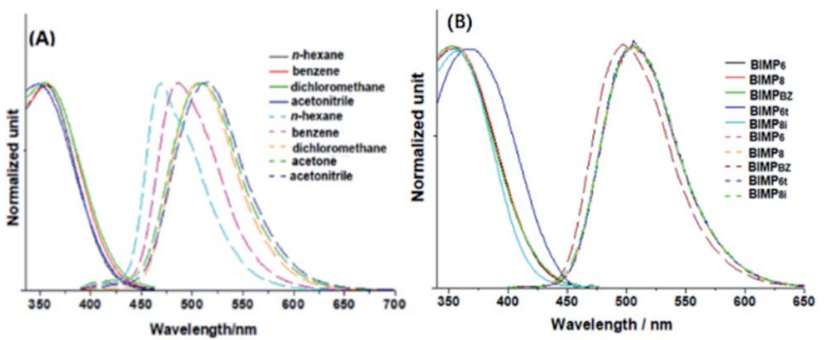

Fig. 2 Absorption (solid lines) and emission spectra (dashed lines) of $\mathrm{BIMP}_{2}$ in different solvents (A); absorption (solid lines) and emission spectra (dashed lines) of BIMP members in dichloromethane solution (B).

slightly dependent on the solvent polarity, while the emission wavelength $\left(\lambda_{\mathrm{em}}\right)$ maxima are sensitive to it. As the polarity increases, $\lambda_{\mathrm{em}}$ are gradually red-shifted and a positive solvatochromic effect is produced. However, the degree of such an effect is not so large that a slight shift cannot be further generated in a solvent whose polarity is higher than that of dichloromethane. This result indicates that an intramolecular charge transfer (ICT) should occur in the excited state, however, the extent of such a transfer is not great. Additionally, it was found that the absorption and the emission band in each solvent were almost completely isolated with little overlap, which implies that the excited state experiences a considerably geometric relaxation before going back to the ground state. Actually, the rotations of 4-cyanophenyl and PTZ around the benzimidazole core along with the flipping of the PTZ ring and the pendulation of the ethyl group facilitate the dissipation of excitation energy and the emergence of a very large Stoke's shift. The similar absorption and emission behaviours in solution also take place on other BIMP members (Fig. S1-S6, ESI $\dagger$ ).

The absorption and emission spectra of BIMP members in dichloromethane were surveyed (Fig. 2B), which indicated that the $\lambda_{\mathrm{ab}}$ of all BIMP fluorophores with alkyl chains were almost identical, likewise their $\lambda_{\mathrm{em}}$. This means that alkyl chains have no influence on the photophysical properties of these compounds in the isolated state. For $\mathbf{B I M P}_{\mathbf{B Z}}$, the chain effect of the phenyl group is slightly different from those of alkyls, since the $\lambda_{\mathrm{ab}}$ and the $\lambda_{\mathrm{em}}$ of $\mathbf{B I M P} \mathbf{P}_{\mathbf{B Z}}$ cause a slight red and blue shift, respectively, as compared with those of other BIMP members.

\section{X-ray crystallography}

Sharply different from the isolated state, the physical properties of fluorophores in the solid-state are closely related to conformations and intermolecular interactions. Hence, BIMP members with different side chains will certainly have distinct optical features and MFC behaviors. In order to explore the structure-property relationship between the side chain and emission wavelength in the pristine state, we firstly surveyed the crystalline structures of these homologues in view of packing density, molecular conformations (Table 1) and intermolecular interactions (Table 2).

The single crystals of BIMP members can be grown from an $n$-hexane/ $\mathrm{CHCl}_{3}$ binary mixture by slow evaporation in air. X-ray 
Table 1 Dihedral angles $(\theta)$, bond angles $(\phi)$ and packing density $(D)$ of BIMP members in the crystalline phase

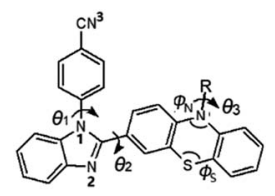

\begin{tabular}{llllrll}
\hline Compd & $\theta_{1}\left(^{\circ}\right)$ & $\theta_{2}\left(^{\circ}\right)$ & $\theta_{3}\left(^{\circ}\right)$ & $\Phi_{\mathrm{N}}\left(^{\circ}\right)$ & $\Phi_{\mathrm{S}}\left(^{\circ}\right)$ & $D\left(\mathrm{~g} \mathrm{~cm}^{-3}\right)$ \\
\hline BIMP $_{\mathbf{1}}$ & 47.43 & 31.55 & - & 98.01 & 118.19 & 1.351 \\
BIMP $_{2}$ & 51.94 & 54.33 & - & 98.56 & 120.03 & 1.329 \\
BIMP $_{\mathbf{6}}$ & 50.21 & 41.74 & - & 98.60 & 118.27 & 1.277 \\
BIMP $_{6 \mathbf{6 t}}$ & 51.45 & 36.89 & - & 96.91 & 116.32 & $-{ }^{\circ}$ \\
BIMP $_{\mathbf{8 i}}$ & 61.82 & 23.56 & - & 97.58 & 119.04 & 1.223 \\
BIMP $_{\mathbf{8}}$ & 62.41 & 15.14 & - & 96.64 & 115.45 & 1.207 \\
BIMP $_{\text {BZ }}$ & 52.02 & 68.83 & 74.87 & 100.70 & 122.27 & 1.374
\end{tabular}

${ }^{a}$ Density cannot be precisely obtained as the crystal contains solvent molecules.

crystallography demonstrates that no $\mathrm{C}-\mathrm{H} \cdots \pi$ interactions emerge in each tested crystal (Fig. 3, S7, S8 $\dagger$ and 6A). Except for the $\mathbf{B I M P}_{\mathbf{B Z}}$ crystal, no $\pi \cdots \pi$ interactions can be found in other BIMP crystals with alkyl chains, where conformations are fixed by $\mathrm{C}-\mathrm{H} \cdots \mathrm{N}$ and $\mathrm{C}-\mathrm{H} \cdots \mathrm{S}$ interactions along with van der Waals forces. This indicates very good accordance between the packing density and the conformation distortion. In BIMP crystals with alkyl chains except $\mathbf{B I M P}_{\mathbf{1}}$, the conformations gradually become more twisted as the packing density goes up, when the sum of the two dihedral angles $\theta_{1}$ and $\theta_{2}$ is considered. In $\mathbf{B I M P}_{2}, \mathbf{B I M P}_{\mathbf{6}}$ and $\mathbf{B I M P}_{\mathbf{8}}$ crystals, the free volumes are drastically improved due to the extension of the unbranched alkyl chains, which permit the molecules to produce less twisted conformations. In the $\mathbf{B I M P}_{\mathbf{8 i}}$ crystal, there is a larger packing density and a more twisted conformation as compared to the $\mathbf{B I M P}_{\mathbf{8}}$ crystal, since the reduced free volume triggered by the shortening of the chain length is unable to be fully offset by the improved one due to branching of the side chain. In the BIMP $_{6 \mathbf{t}}$ crystal, however, the highly branched 2,2-dimethylbutyl chain leads to the denser packing and more twisted conformation than in the $\mathbf{B I M P}_{\mathbf{6}}$ crystal, owing to the considerably increased free volume that effectively conquers the reduced one

Table 2 Intermolecular interactions in crystals of BIMP members

\begin{tabular}{llllll}
\hline Compd & $\mathrm{C}-\mathrm{H} \cdots \mathrm{N}_{1}(\AA)$ & $\mathrm{C}-\mathrm{H} \cdots \mathrm{N}_{2}(\AA)$ & $\mathrm{C}-\mathrm{H} \cdots \mathrm{N}_{3}(\AA)$ & $\mathrm{C}-\mathrm{H} \cdots \mathrm{S}(\AA)$ & $\pi-\pi(\AA)$ \\
\hline BIMP $_{\mathbf{1}}$ & - & 2.674 & 2.506 & $3.242^{a}$ & - \\
BIMP $_{2}$ & 3.357 & 2.778 & 2.876 & 3.102 & - \\
BIMP $_{6}$ & - & - & 2.657 & 3.458 & - \\
BIMP $_{6 \mathbf{t}}$ & 3.100 & - & 2.706 & 3.011 & - \\
BIMP $_{\mathbf{8 i}}$ & - & 2.550 & - & 3.260 & - \\
BIMP $_{\mathbf{8}}$ & - & 2.687 & - & 3.559 & - \\
BIMP $_{\mathbf{B Z}}$ & 2.955 & - & 2.589 & 3.086 & 3.417
\end{tabular}

${ }^{a}$ This H-bond is intramolecular.
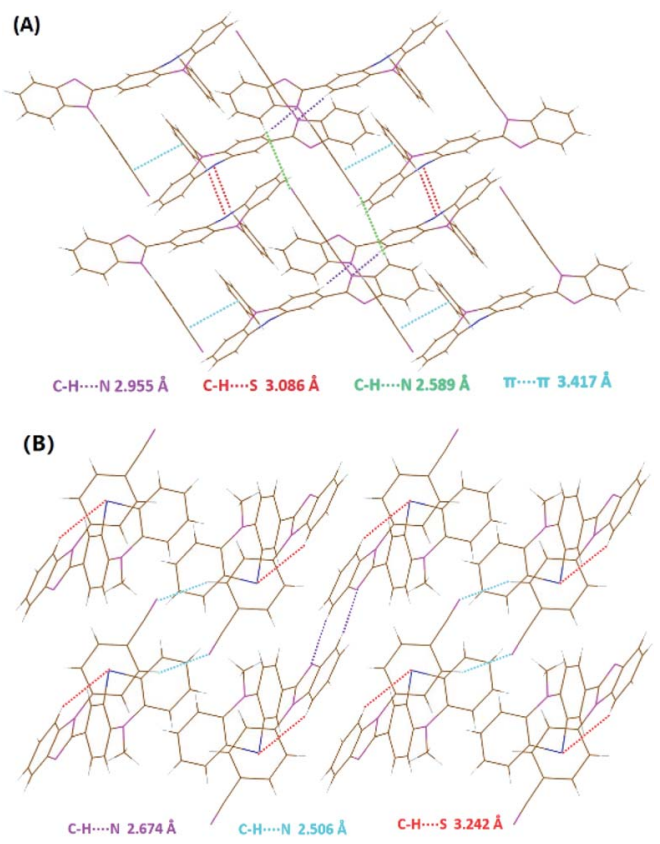

Fig. 3 Molecular packing of $\mathrm{BIMP}_{\mathrm{BZ}}(\mathrm{A})$ and $\mathrm{BIMP}_{1}(\mathrm{~B})$.

induced by the shortening of the alkyl chain length. These results indicate that both the length and branching degree of a side chain should be considered when MFC-tunable systems are designed, and it seems that the latter has a greater impact on conformations in the crystalline phase than the former.

Although the packing density in the $\mathbf{B I M P}_{\mathbf{1}}$ crystal is the largest for BIMP members with alkyl chains, the conformation is abnormally less twisted with smaller dihedral angles. Unlike all the other BIMP crystals, where the alkyl chains on PTZ and the $\mathrm{C}=\mathrm{N}$ bonds on imidazoles are located at both sides of the $\sigma$ bond linking the PTZ moiety and the imidazole ring, the methyl chain and $\mathrm{C}=\mathrm{N}$ bond are on the same side in the $\mathbf{B I M P}_{\mathbf{1}}$ crystal, which results in intramolecular $\mathrm{C}-\mathrm{H} \cdots \mathrm{S}$ interactions. These effectively reduce the free volume and make the less twisted conformation preferable, even in dense packing (Fig. 3B). In the BIMP $_{\mathbf{B Z}}$ crystal (Fig. $3 \mathrm{~A}$ ), the strong $\pi \cdots \pi$ interaction (3.417 $\AA$ ) between the phenyl chain and 4-cyanophenyl considerably shortens the distance between adjacent molecules. Moreover, the H-bonds in this crystal are remarkably stronger than those in the $\mathbf{B I M P}_{2}$ crystal where the conformation is the most twisted among BIMP homologues with alkyl chains. Therefore, the shorter $\mathrm{H}$-bonds together with the strong $\pi \cdots \pi$ interactions cause the $\mathbf{B I M P}_{\mathbf{B z}}$ crystal to have the largest packing density and the highest conformation distortion in the BIMP family. Additionally, only very slight changes in bond angles $\phi_{\mathrm{N}}$ and $\phi_{\mathrm{S}}$ occur on the PTZ moiety in BIMP crystals with different side chains, which indicates that the conformational differences in these crystals should be triggered by the side chain effect instead of the opening and closing of the PTZ ring skeleton. 


\section{Chain-dependent solid-state physical property}

It is well known that the emission behaviours of crystals are closely related to molecular conformations and intermolecular interactions. For homologues with similar types of intermolecular interactions, their emission performances are dominantly dependent on molecular conformations. Hence, the structureproperty study in this BIMP family should be focused on conformations in the pristine state. The emission wavelengths of BIMP samples before and after force stimuli are listed in Table 3 .

For BIMP homologues with alkyl chains, it has been demonstrated that there is good accordance between emission wavelengths and molecular conformations in the crystalline phase. As the conformational distortion is gradually reduced, the emission wavelength of the corresponding crystal is also progressively red-shifted because smaller dihedral angles can cause molecules to contain larger $\pi$-conjugated area. Except for BIMP $_{1}$ with the special orientation of the PTZ group, the dihedral angle $\theta_{2}$ is greatly reduced but $\theta_{1}$ is not monotonically varied as the length and branching degree of alkyl chains are enhanced. Overall, compared with the considerable alteration in $\theta_{2}$, there was only a slight change in $\theta_{1}$. Hence, it appears that it is $\theta_{2}$ that has a dominant influence on the emission wavelength of the corresponding crystal. For BIMP members with alkyls, except for $\mathbf{B I M P}_{\mathbf{8 i}}$, it was observed that the emission wavelengths of the ground samples were almost identical. We assume that hand grinding can just destroy lattices and planarize the conformations but it is not strong enough to compress the distances between adjacent molecules, which are sensitive to the length and branching degree of the side chains. It has been verified that only GPa-order high pressure can shorten such distances. ${ }^{18}$ Consequently, the ground BIMP samples with alkyl chains present almost identical emission wavelengths, since alkyls make very little contribution to the $\pi$ conjugation on the planarized molecules. However, the $\mathbf{B I M P}_{\mathbf{8 i}}$ ground sample displays the shorter emission wavelength and the reason is unknown at the present time. Although $\mathbf{B I M P}_{\mathbf{B Z}}$ molecules have the most twisted conformations among all BIMP members, the emission wavelength of the $\mathbf{B I M P}_{\mathbf{B Z}}$ crystal is remarkably red-shifted as compared to that of the $\mathbf{B I M P}_{\mathbf{2}}$ crystal in which the conformation is the most twisted among BIMP homologues with alkyl chains. This indicates that the redshifted emission triggered by the dissipation of excited energy due to the strong $\pi-\pi$ interaction in the $\mathbf{B I M P}_{\mathbf{B Z}}$ crystal

Table 3 Emission wavelengths of BIMP members under different conditions

\begin{tabular}{llllllll}
\hline Compd & $\begin{array}{l}\lambda_{\text {cry }} \\
(\mathrm{nm})\end{array}$ & $\begin{array}{l}\lambda_{\text {am }} \\
(\mathrm{nm})\end{array}$ & $\Delta \lambda^{a}(\mathrm{~nm})$ & Compd & $\begin{array}{l}\lambda_{\text {cry }} \\
(\mathrm{nm})\end{array}$ & $\begin{array}{l}\lambda_{\text {am }} \\
(\mathrm{nm})\end{array}$ & $\Delta \lambda^{a}(\mathrm{~nm})$ \\
\hline $\mathbf{B I M P}_{\mathbf{1}}$ & 475 & 503 & 28 & $\mathbf{B I M P}_{\mathbf{8}}$ & 490 & 502 & 12 \\
$\mathbf{B I M P}_{2}$ & 453 & 503 & 50 & $\mathbf{B I M P}_{\mathbf{8 i}}$ & 480 & 490 & 10 \\
$\mathbf{B I M P}_{\mathbf{6}}$ & 467 & 502 & 35 & $\mathbf{B I M P}_{\mathbf{B Z}}$ & 476 & 513 & 37 \\
BIMP $_{\mathbf{6 t}}$ & 471 & 501 & 30 & & & &
\end{tabular}

${ }^{a} \Delta \lambda=\lambda_{\text {am }}-\lambda_{\text {cry }}$ effectively overcomes the blue-shifted one induced by highly conformational distortion. Under force stimuli, the dihedral angle $\theta_{3}$ between the phenyl chain and the PTZ ring may also be greatly reduced, causing the $\pi$-conjugated areas on $\mathbf{B I M P}_{\mathbf{B Z}}$ molecules to be extended and the emission wavelength in amorphous phase to be red-shifted.

Powder X-ray diffraction (PXRD) demonstrates that the MFC activity of BIMP members could be attributed to the dynamically reversible morphological transformations between the crystalline and amorphous phases (Fig. S9-S15, ESI $\dagger$ ). The PXRD pattern of each as-prepared sample is almost identical to that simulated by X-ray crystallography, which means that each pristine sample has the same crystalline structure as the corresponding single crystal. By solvent fuming or annealing, the highly dispersed bands on PXRD patterns of ground samples can be completely restored to the original sharp and intense peaks of the as-prepared ones. At the same time, the emission spectra of ground samples can be fully recovered to those of asprepared ones (Fig. 4 and S16-S18, ESI $\dagger$ ). However, the emission behavior and PXRD pattern of the ground $\mathbf{B I M P}_{\mathbf{6 t}}$ sample can only be reproduced into those of the pristine ones by fuming in $\mathrm{CHCl}_{3}$. X-ray crystallography indicates that equimolar ratios of $\mathbf{C H C l}_{3}$ molecules are locked in the caves of the $\mathbf{B I M P} \mathbf{P}_{\mathbf{6 t}}$ crystal by strong $\mathrm{H}$-bonds between $\mathrm{N}_{\text {imidazole }}$ and $\mathrm{H}_{\mathrm{CHCl}_{3}}$ (Fig. S8A, ESI $\dagger$ ). Due to the irregularity and the intensely thermal motion of 2,2-dimethylbutyl, the $\mathbf{B I M P}_{\mathbf{6 t}}$ molecules in the ground sample where $\mathrm{CHCl}_{3}$ is released from broken lattices can hardly be packed into the original crystalline structure by short-time annealing without a $\mathrm{CHCl}_{3}$ atmosphere.

Moreover, the thermal properties of BIMP members are tested by differential scanning calorimetry (DSC). On the DSC curves of all ground samples except $\mathbf{B I M P}_{\mathbf{6 t}}$, the exothermal peaks (cold crystallization) can clearly be observed (Fig. S19†). This indicates that the repeat of the original emission behaviours by annealing treatment could be ascribed to cold
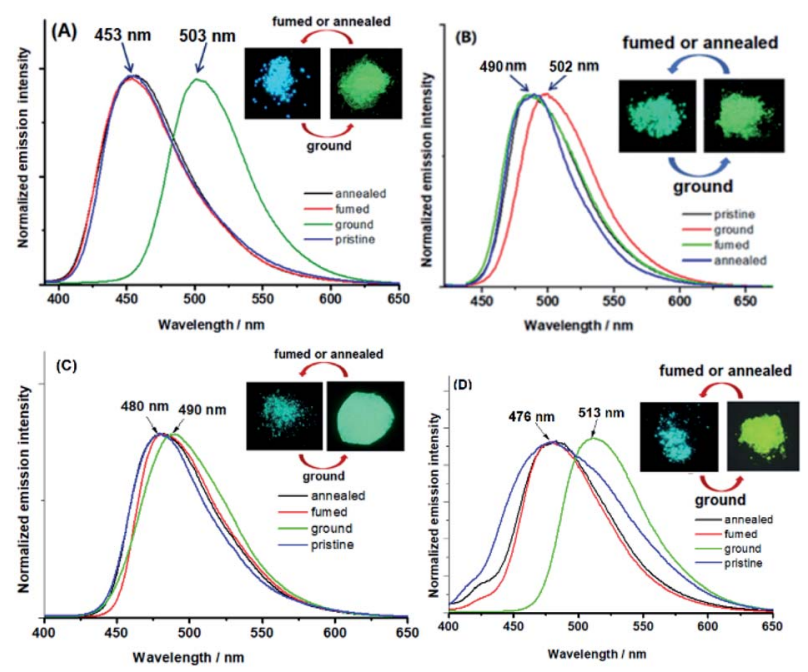

Fig. 4 Solid-state emission spectra of $\mathrm{BIMP}_{2}(\mathrm{~A}), \mathrm{BIMP}_{8}(\mathrm{~B}), \mathrm{BIMP}_{8 \mathrm{i}}(\mathrm{C})$ and $B I M P_{B Z}(D)$ under different conditions (inset: photos of samples taken under $365 \mathrm{~nm}$ UV light). 
crystallization. Conformational changes from the crystalline phase to the amorphous phase will produce inner stress in ground samples, which can be mostly or fully relieved under heat treatment, therefore, the temperature of cold crystallization indicates the strength of the inner stress. Under vigorous grinding, the lattices are completely destroyed and the conformations may well be entirely planarized. Hence, the temperature of cold crystallization $\left(T_{\mathrm{CC}}\right)$ implies the extent of conformational distortion in the crystalline phase. Overall, the level of $T_{\mathrm{CC}}$ is in good accordance with the degree of conformational distortion in this BIMP family. The $T_{\mathrm{CC}}$ of $\mathbf{B I M P}_{\mathbf{B Z}}$ is the highest $\left(114.7^{\circ} \mathrm{C}\right)$ while the $T_{\mathrm{CC}}$ of $\mathbf{B I M P}_{\mathbf{8}}$ is the lowest $(70.0$ $\left.{ }^{\circ} \mathrm{C}\right)$. Moreover, the $T_{\mathrm{CC}}$ of $\mathbf{B I M P}_{\mathbf{8 i}}\left(85.8^{\circ} \mathrm{C}\right)$ is higher than those of BIMP $_{6}\left(89.2{ }^{\circ} \mathrm{C}\right)$ and $\mathbf{B I M P}_{2}\left(98.7^{\circ} \mathrm{C}\right)$, as the former has the less twisted conformation than the latter. Although the extent of the conformational distortion of $\mathbf{B I M P}_{\mathbf{1}}$ and $\mathbf{B I M P}_{\mathbf{8}}$ is very close to each other, the $T_{\mathrm{CC}}$ of the former $\left(84.3{ }^{\circ} \mathrm{C}\right)$ is remarkably higher than that of the latter. It is presumed that the inner stress from intramolecular $\mathrm{H}_{\mathrm{s}}$-bonds in the BIMP $\mathbf{P}_{\mathbf{1}}$ sample may consume more heat energy to be relieved. For the ground BIMP $_{6 \mathbf{t}}$ sample, neither cold crystallization nor melting peaks can be observed on the DSC curve and it is gradually upraised as the temperature increases, which is characteristic of an amorphous sample. Additionally, it was shown that the melting temperatures of $\mathbf{B I M P}_{\mathbf{1}}, \mathbf{B I M P}_{\mathbf{2}}$ and $\mathbf{B I M P}_{\mathbf{B Z}}$ crystals are considerably higher than those of $\mathbf{B I M P}_{6}, \mathbf{B I M P}_{\mathbf{8}}$ and $\mathbf{B I M P}_{\mathbf{8 i}}$, as a larger packing density means stronger intermolecular interactions and more stable lattices that have to be destroyed at higher temperatures.

\section{Mechanoluminescence effect on BIMP $_{6}$}

Mechanoluminescence (ML), also known as triboluminescence, is a kind of unique luminescence triggered by mechanical excitation (motion or stress force) of solid materials, which was firstly discovered by Francis Bacon in 1605. Unlike a great deal of MFC-active systems, ML-active systems composed of purely organic molecules are relatively limited..$^{19}$ Although there are a few cases where centrosymmetric crystals exhibit excellent ML effects, ${ }^{20}$ most reported ML-active crystals still have noncentrosymmetric or even chiral space groups. It is generally believed that ML activity may well originate from the piezoelectric effect, and non-centrosymmetric crystals are preferable for producing electron discharge due to the non-zero net dipole moment under the force-induced separation of amphoteric charges. Such discharge can excite molecules on and very close to freshly cracked surfaces to produce luminescence. Certainly, it is reasonable that both the high discharge strength caused by large net dipole moments and heavy discharge quantities provided by enhanced discharge surface areas are helpful for crystals to present excellent ML performance.

It is interesting to find that the $\mathbf{B I M P}_{\mathbf{6}}$ crystal is highly MLactive; a very brightly greenish-yellow flashlight can be clearly seen when the crystal is lightly pressed with a stainless steel spatula (Fig. 5B). Sharply different from most other purely organic ML-active molecules whose luminescence can only be observed in the dark by application of heavy force, the ML effect of the $\mathbf{B I M P}_{\mathbf{6}}$ crystal is so intense that luminescence can be readily seen even under daylight conditions (Fig. 5C). It has been demonstrated that the emission wavelength $(498 \mathrm{~nm})$ on the ML spectrum is very close to that on the PL spectrum (502 $\mathrm{nm}$ ) of the ground sample (Fig. 5A). Such similarity implies that the $\mathbf{B I M P}_{\mathbf{6}}$ crystal is very readily broken into amorphous particles on exposure to force stimuli while the photo- or the forceinduced emission may well be derived from the same excitedstate species.

X-ray crystallography indicates that $\mathbf{B I M P}_{\mathbf{6}}$ molecules are packed into a crystal with a monoclinic system and $P 2_{1}$ chiral space group. Intriguingly, such a chiral crystal is constructed by molecules without any chiral units. It has been demonstrated that only two types of $\mathrm{H}$-bonds $\left(\mathrm{C}-\mathrm{H} \cdots \mathrm{N}_{\text {cyano }} 2.657 \AA\right.$ and $\mathrm{C}-\mathrm{H} \cdots \mathrm{S} 3.458 \AA$ ) emerge in the BIMP $_{6}$ crystal without $\pi-\pi$ and $\mathrm{C}-\mathrm{H} \cdots \pi$ interactions (Fig. 6A). These H-bonds arrange the molecules into one-dimensional chains on the $b-c$ plane and very slight interactions occur among chains. In each chain, the adjacent molecules are respectively drawn together by $\mathrm{N}-\mathrm{H}$ and S-H bonds to form couple I and II (Fig. 6C). Moreover, little interactions appear among molecules along the $a$-axis. Hence, the crystal is extremely fragile and readily splits along the directions of packed chains and $a$-axis with the application of external force (Fig. 6B) to produce many fresh surfaces. It has been reported that the analysis of net dipole moments on couples could properly interpret the reason for ML activity for a crystal; $;{ }^{20 b}$ we carried out the DFT calculations at the B3LYP/6$31 \mathrm{G}(\mathrm{d}, \mathrm{p})$ level based on the conformations in the crystalline phase. It has been demonstrated that the dipole moment of an individual molecule is just 1.886 Debye, while the net dipole moments of couple I and II are 3.688 and 1.548 Debye (Fig. 6D), respectively. Therefore, the non-zero net dipole moments can trigger discharges between couples on those freshly cracked surfaces under external force, which excites the molecules on such surfaces either by electron impact or ionization followed by electron-hole recombination, similar to electroluminescence. However, these discharges are not strong enough to result in the intense ML effect as the net dipole moments of couples are still relatively small. Owing to the particular chain packing in the $\mathbf{B I M P}_{\mathbf{6}}$ crystal, so many fresh surfaces can be readily generated to provide largely increased discharge areas when the crystal is exposed to force stimuli, on which heavy discharge quantities can be offered and may well be mainly responsible for the excellent ML activity of this crystal.

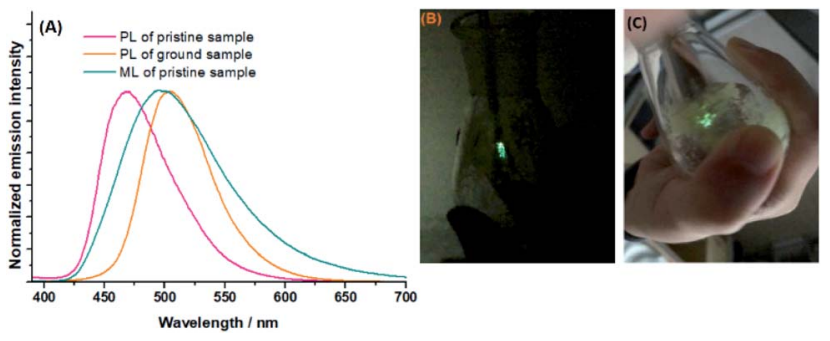

Fig. $5 \mathrm{PL}$ and $\mathrm{ML}$ spectra of $\mathrm{BIMP}_{6}$ samples $(\mathrm{A})$; photos of the $\mathrm{ML}$ phenomenon on a $\mathrm{BIMP}_{6}$ crystal taken in the dark (B) and in daylight (C). 
(A)

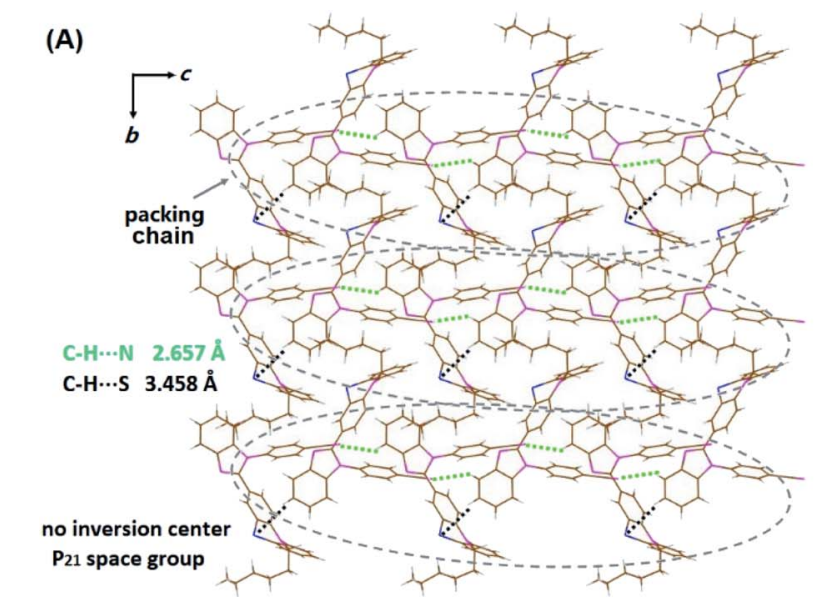

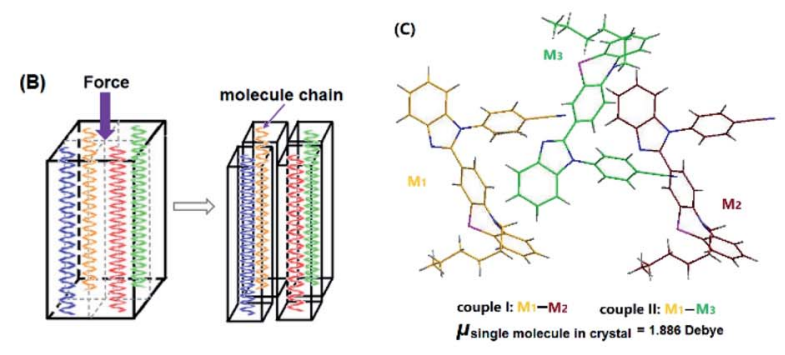

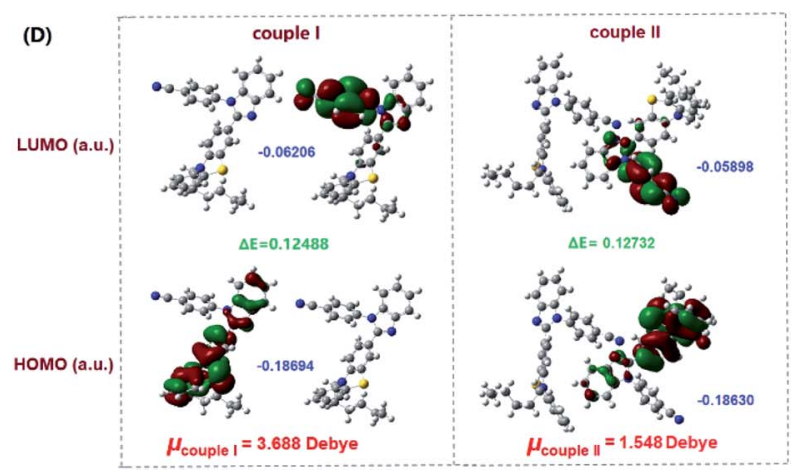

Fig. 6 Molecular packing in the $\mathrm{BIMP}_{6}$ crystal (A) and schematic diagram of the crystal before and after force stimuli (B); couples in this crystal (C) and calculated energy levels and electron distribution on the HOMO and LUMO of molecules in these couples (D).

Meanwhile, the great fragility of the crystal leads to the quite easy transformation from the large-sized particles into the tiny ones, which makes the ML spectrum very close to the PL one in the amorphous phase.

\section{Conclusion}

We have obtained a family of PTZ-substituted benzimidazoles with different side chains and carried out detailed structureproperty research regarding MFC performance and molecular conformations in the crystalline phase. It has been demonstrated that conformation analysis is a suitable way to reveal the intrinsic solid-state emission behaviours of homologues with similar types of intermolecular interactions and packing modes. Overall, for BIMP homologues with alkyl chains, the emission wavelengths of their pristine samples are gradually red-shifted due to the progressively diminished extent of conformation distortion as the length or the branching degree of alkyl chains is increased, while the MFC activity is correspondingly reduced due to emission wavelengths in the amorphous phase that are almost the same. The intramolecular S-H bonds in BIMP $_{1}$ crystals caused by the unique orientation of PTZ can account for the unexpectedly less twisted conformation. Although the densest packing appears in the $\mathbf{B I M P}_{\mathbf{B Z}}$ crystal with the most twisted conformations, the strong $\pi-\pi$ interactions make a great contribution to the remarkably redshifted emission wavelength. Meanwhile, the enlarged $\pi$ conjugation area generated by the planarization of the phenyl chain under force stimuli causes the emission wavelength of the ground $\mathbf{B I M P}_{\mathbf{B z}}$ sample to be more red-shifted as compared to those of other ground BIMP samples. Additionally, the MFC activity of this family can be ascribed to morphological changes and the cold crystallization temperature is in good accordance with the degree of conformational distortion. X-ray crystallography and DFT calculations reveal that the very bright luminescence from the ML effect on $\mathbf{B I M P}_{6}$ crystals can mainly be attributed to large discharge quantity rather than high discharge strength due to the extreme fragility of this crystal caused by particular chain packing.

\section{Conflicts of interest}

There are no conflicts to declare.

\section{Acknowledgements}

We are grateful for financial support from the Natural Science Fund of Zhejiang Province (LY19B020015). We also thank Prof. Zhenguo Chi and Dr Zongliang Xie very much for their kindly help on ML spectra measurements. We thank Dr Jiyong Liu very much for his kindly help on X-ray crystallographic measurements and analysis.

\section{Notes and references}

1 (a) Y. Q. Dong, J. W. Y. Lam and B. Z. Tan, J. Phys. Chem. Lett., 2015, 6, 3429-3436; (b) Z. Chi, X. Zhang, B. Xu, X. Zhou, C. Ma, Y. Zhang, S. Liu and J. Xu, Chem. Soc. Rev., 2012, 41, 3878-3896; (c) C. Wang and Z. Li, Mater. Chem. Front., 2017, 1, 2174-2194; (d) Z. Yang, Z. Chi, Z. Mao, Y. Zhang, S. Liu, J. Zhao, M. P. Aldred and Z. Chi, Mater. Chem. Front., 2018, 2, 861-890; (e) S. Varughese, J. Mater. Chem. $C$, 2014, 2, 3499-3516; (f) Y. Sagara, S. Yamane, M. Mitani, C. Weder and T. Kato, Adv. Mater., 2016, 28, 1073-1095.

2 (a) P. Shi, Y. Duan, W. Wei, Z. Xu, Z. Li and T. Han, J. Mater. Chem. C, 2018, 6, 2476-2482; (b) C. Feng, K. Wang, Y. Xu, L. Liu, B. Zou and P. Lu, Chem. Commun., 2016, 52, 38363839; (c) L. Wang, K. Wang, B. Zou, K. Ye, H. Zhang and Y. Wang, Adv. Mater., 2015, 27, 2918-2922; (d) W.-Z. Xie, H.-C. Zheng and Y.-S. Zheng, J. Mater. Chem. C, 2017, 5, 10462-10468. 
3 (a) S. Hirata and T. Watanabe, Adv. Mater., 2006, 18, 27252729; (b) S. J. Lim, B. K. An, S. D. Jung, M. A. Chung and S. Y. Park, Angew. Chem., Int. Ed., 2004, 43, 6346-6350; (c) C. E. Olson, M. J. R. Previte and J. T. Fourkas, Nat. Mater., 2002, 1, 225-228; (d) M. Irie, T. Fukaminato, T. Sasaki, N. Tamai and T. Kawai, Nature, 2002, 420, 759-760.

4 (a) A. Kishimura, T. Yamashita, K. Yamaguchi and T. Aida, Nat. Mater., 2005, 4, 546-549; (b) X. Zhu, R. Liu, Y. Li, H. Huang, Q. Wang, D. Wang, X. Zhu, S. Liu and H. Zhu, Chem. Commun., 2014, 50, 12951-12954; (c) Q. Qi, Y. Liu, X. Fang, Y. Zhang, P. Chen, Y. Wang, B. Yang, B. Xu, W. Tian and S. X.-A. Zhang, RSC Adv., 2013, 3, 7996-8002; (d) P. Kumar, J. Dwivedi and B. K. Gupta, J. Mater. Chem. C, 2014, 2, 10468-10475; (e) X.-L. Lu and M. Xia, J. Mater. Chem. C, 2016, 4, 9350-9358.

5 (a) W. Z. Yuan, Y. Tan, Y. Gong, P. Lu, J. W. Y. Lam, X. Y. Shen, C. Feng, H. Y. Sung, Y. Lu, I. D. Williams, J. Z. Sun, Y. Zhang and B. Z. Tang, Adv. Mater., 2013, 25, 2837-2843; (b) C. Li, X. Tang, L. Zhang, C. Li, Z. Liu, Z. Bo, Y. Q. Dong, Y.-H. Tian, Y. Dong and B. Z. Tang, Adv. Opt. Mater., 2015, 3, 1184-1190; (c) K. C. Naeem, A. Subhakumari, S. Varughese and V. C. Nair, J. Mater. Chem. C, 2015, 3, 10225-10231; (d) J. Sun, J. Han, Y. Liu, Y. Duan, T. Han and J. Yuan, J. Mater. Chem. C, 2016, 4, 8276-8283; (e) P. Xue, Z. Yang and P. Chen, J. Mater. Chem. $C, 2018,6,4994-5000$.

6 (a) Y. Sagara and T. Kato, Angew. Chem., Int. Ed., 2011, 50, 9128-9132; (b) S.-J. Yoon, J. W. Chung, J. Gierschner, K. S. Kim, M.-G. Choi, D. Kim and S. Y. Park, J. Am. Chem. Soc., 2010, 132, 13675-13683; (c) H. Sun, S. Liu, W. Lin, K. Y. Zhang, W. Lv, X. Huang, F. Huo, H. Yang, G. Jenkins, Q. Zhao and W. Huang, Nat. Commun., 2014, 5, 3601-3609; (d) K. Y. Zhang, S. Liu, Q. Zhao and W. Huang, Coord. Chem. Rev., 2016, 319, 180-195; (e) X. Chen, G. Sun, T. Zhang, S. Liu, Q. Zhao and W. Huang, Adv. Mater., 2016, 28, 7137-7142; $(f)$ Q. Zhao, W. Xu, H. Sun, J. Yang, K. Y. Zhang, S. Liu, Y. Ma and W. Huang, Adv. Opt. Mater., 2016, 4, 1167-1173; (g) W. Lin, Q. Zhao, H. Sun, K. Y. Zhang, H. Yang, Q. Yu, X. Zhou, S. Guo, S. Liu and W. Huang, Adv. Opt. Mater., 2015, 3, 368-375; (h) J. Han, J. Sun, Y. Li, Y. Duan and T. Han, J. Mater. Chem. C, 2016, 4, 9287-9293.

7 (a) S. Xue, X. Qiu, Q. Sun and W. Yang, J. Mater. Chem. C, 2016, 4, 1568-1578; (b) X. Huang, L. Qian, Y. Zhou, M. Liu, Y. Cheng and H. Wu, J. Mater. Chem. C, 2018, 6, 5075-5096.

8 (a) Y. Liu, Y. Lei, F. Li, J. Chen, M. Liu, X. Huang, W. Gao, H. Wu, J. Ding and Y. Cheng, J. Mater. Chem. C, 2016, 4, 2862-2870; (b) Y. Wang, W. Liu, L. Bu, J. Li, M. Zheng, D. Zhang, M. Sun, Y. Tao, S. Xue and W. Yang, J. Mater. Chem. C, 2013, 1, 856-862; (c) L. Bu, M. Sun, D. Zhang, W. Liu, Y. Wang, M. Zheng, S. Xue and W. J. Yang, J. Mater. Chem. C, 2013, 1, 2028-2035; (d) M. Zheng, D. T. Zhang, M. X. Sun, Y. P. Li, T. L. Liu, S. F. Xue and W. J. Yang, J. Mater. Chem. C, 2014, 2, 1913-1920.

9 (a) Y. Wang, W. Liu, L. Bu, J. Li, M. Zheng, D. Zhang, M. Sun, Y. Tao, S. Xue and W. Yang, J. Mater. Chem. C, 2013, 1, 856-
862; (b) Q. K. Sun, W. Liu, S. A. Ying, L. L. Wang, S. F. Xue and W. J. Yang, RSC Adv., 2015, 5, 73046-73050.

10 L. Y. Bu, Y. P. Li, J. F. Wang, M. X. Sun, M. Zheng, W. Liu, S. F. Xue and W. J. Yang, Dyes Pigm., 2013, 99, 833-838.

11 P. Xue, B. Yao, X. Liu, J. Sun, P. Gong, Z. Zhang, C. Qian, Y. Zhang and R. Lu, J. Mater. Chem. C, 2015, 3, 1018-1025.

12 N. D. Nguyen, G. Q. Zhang, J. W. Lu, A. E. Sherman and C. L. Fraser, J. Mater. Chem., 2011, 21, 8409-8415.

13 (a) P. Xue, B. Yao, X. Liu, J. Sun, P. Gong, Z. Zhang, C. Qian, Y. Zhang and R. Lu, J. Mater. Chem. C, 2015, 3, 1018-1025; (b) L. Qian, Y. Zhou, M. Liu, X. Huang, G. Wu, W. Gao, J. Ding and $\mathrm{H}$. Wu, RSC Adv., 2017, 7, 42180-42191.

14 (a) Z.-Y. Wang, J.-W. Zhao, P. Li, T. Feng, W.-J. Wang, S.-L. Tao and Q.-X. Tong, New J. Chem., 2018, 42, 89248932; (b) T. Jadhav, J. M. Choi, B. Dhokale, S. M. Mobin, J. Y. Lee and R. Misra, J. Phys. Chem. C, 2016, 120, 1848718495; (c) T. Jadhav, J. M. Choi, J. Shinde, J. Y. Lee and R. Misra, J. Mater. Chem. C, 2017, 5, 6014-6020; (d) T. Jadhav, B. Dhokale, S. M. Mobin and R. Misra, J. Mater. Chem. C, 2015, 3, 9981-9988; (e) A. Ekbote, S. H. Han, T. Jadhav, S. M. Mobin, J. Y. Lee and R. Misra, J. Mater. Chem. C, 2018, 6, 2077-2087; (f) R. Misra, T. Jadhav, B. Dhokale and S. M. Mobin, Chem. Commun., 2014, 50, 9076-9078; $(g)$ Y. Zhan, Y. Xu, Z. Jin, W. Ye and P. Yang, Dyes Pigm., 2017, 140, 452-459; (h) Z. Gao, K. Wang, F. Liu, C. Feng, X. He, J. Li, B. Yang, B. Zou and P. Lu, Chem.-Eur. J., 2017, 23, 773-777; (i) Y. Cai, Y.-M. Yin, H.-T. Cao, M. Zhang, G.-G. Shan, H.-Z. Su, Y. Wu, Z.-M. Su and W.-F. Xie, Dyes Pigm., 2015, 119, 62-69.

15 (a) B. Huang, W.-C. Chen, Z. Li, J. Zhang, W. Zhao, Y. Feng, B. Z. Tang and C.-S. Lee, Angew. Chem., Int. Ed., 2018, 57, 12473-12477; (b) S. Xiang, Z. Huang, S. Sun, X. Lv, L. Fan, S. Ye, H. Chen, R. Guo and L. Wang, J. Mater. Chem. C, 2018, 6, 11436-11443; (c) B. Xu, Y. Mu, Z. Mao, Z. Xie, H. Wu, Y. Zhang, C. Jin, Z. Chi, S. Liu, J. Xu, Y.-C. Wu, P.-Y. Lu, A. Lien and M. R. Bryce, Chem. Sci., 2016, 7, 22012206; (d) M. Okazaki, Y. Takeda, P. Data, P. Pander, H. Higginbotham, A. P. Monkman and S. Minkata, Chem. Sci., 2017, 8, 2677-2686.

16 (a) J. Zhang, B. Xu, J. Chen, L. Wang and W. Tian, J. Phys. Chem. C, 2013, 117, 23117-23125; (b) A. C. B. Rodrigues, J. Pina, W. Dong, M. Forster, U. Scherf and J. S. Seixas De Melo, Macromolecules, 2018, 51, 8501-8512; (c) C. Dai, D. Yang, W. Zhang, X. Fu, Q. Chen, C. Zhu, Y. Cheng and L. Wang, J. Mater. Chem. B, 2015, 3, 7030-7036; (d) D. Belei, C. Dumea, E. Bicu and L. Marin, RSC Adv., 2015, 5, 8849-8858; (e) L. Wang, Q. Xia, Z. Zhang, J. Qu and R. Liu, Mater. Sci. Eng., 2018, 93, 399-406.

17 (a) Y. Xi, Y. Cao, Y. Zhu, H. Guo, X. Wu, Y. Li and B. Wang, Chem. Lett., 2018, 47, 650-653; (b) K. K. Neena, P. Sudhakar, K. Dipak and P. Thilagar, Chem. Commun., 2017, 53, 3641-3644; (c) Y. Zhan, J. Zhao, P. Yang and W. Ye, RSC Adv., 2016, 6, 92144-92151; (d) G. Zhang, J. Sun, P. Xue, Z. Zhang, P. Gong, J. Peng and R. Lu, J. Mater. Chem. C, 2015, 3, 2925-2932; (e) P. Xue, B. Yao, J. Sun, Q. Xu, P. Chen, Z. Zhang and R. Lu, J. Mater. Chem. C, 2014, 2, 3942-3950. 
18 (a) L. Wang, K. Wang, B. Zou, K. Ye, H. Zhang and Y. Wang, Adv. Mater., 2015, 27, 2918-2922; (b) X. Liu, A. Li, W. Xu, Z. Ma and X. Jia, Phys. Chem. Chem. Phys., 2018, 20, 1324913254.

19 (a) B. Xu, W. Li, J. He, S. Wu, Q. Zhu, Z. Yang, Y.-C. Wu, Y. Zhang, C. Jin, P.-Y. Lu, Z. Chi, S. Liu, J. Xu and M. R. Bryce, Chem. Sci., 2016, 7, 5307-5312; (b) S. Xu, T. Liu, Y. Mu, Y.-F. Wang, Z. Chi, C.-C. Lo, S. Liu, Y. Zhang, A. Lien and J. Xu, Angew. Chem., 2015, 127, 888892; (c) J. Yang, Z. Ren, Z. Xie, Y. Liu, C. Wang, Y. Xie, Q. Peng, B. Xu, W. Tian, F. Zhang, Z. Chi, Q. Li and Z. Li, Angew. Chem., Int. Ed., 2017, 56, 880-884; (d) K. K. Neen,
P. Sudhakar, K. Dipak and P. Thilagar, Chem. Commun., 2017, 53, 3641-3644; (e) B. Xu, J. He, Y. Mu, Q. Zhu, S. Wu, Y. Wang, Y. Zhang, C. Jin, C. Lo, Z. Chi, A. Lien, S. Liu and J. Xu, Chem. Sci., 2015, 6, 3236-3241; (f) H. Nakayama, J. Nishida, N. Takada, H. Sato and Y. Yamashita, Chem. Mater., 2012, 24, 671-676; (g) J. Nishida, H. Ohura, Y. Kita, H. Hasegawa, T. Kawase, N. Takada, H. Sato, Y. Sei and Y. Yamashita, J. Org. Chem., 2016, 81, 433-441.

20 (a) C. Wang, B. Xu, M. Li, Z. Chi, Y. Xie, Q. Lia and Z. Li, Mater. Horiz., 2016, 3, 220-225; (b) F. Liu, J. Tu, X. Wang, J. Wang, Y. Gong, M. Han, X. Dang, Q. Liao, Q. Peng, Q. Li and Z. Li, Chem. Commun., 2018, 54, 5598-5601. 